\title{
Matter-wave interferometry with helium atoms in low- $l$ Rydberg states
}

\author{
J. D. R. Tommey and S. D. Hogan 1 \\ Department of Physics and Astronomy, University College London, Gower Street, London WC1E 6BT, United Kingdom
}

(Received 4 February 2021; revised 7 July 2021; accepted 6 August 2021; published 3 September 2021)

\begin{abstract}
Electric Rydberg-atom interferometry has been performed with helium atoms in coherent superpositions of the $1 s 56 s{ }^{3} S_{1}$ and $1 s 57 s^{3} S_{1}$ Rydberg levels. The experiments were carried out in a longitudinal geometry with the atoms traveling at $2000 \mathrm{~m} / \mathrm{s}$ in pulsed supersonic beams. After laser photoexcitation from the $1 s 2{ }^{3} S_{1}$ metastable level, coherent manipulation of the population of the Rydberg states was achieved using sequences of microwave pulses. The difference in the static electric dipole polarizabilities of the $1 s 56 s{ }^{3} S_{1}$ and $1 s 57 s{ }^{3} S_{1}$ levels allowed superpositions of external momentum states to be generated when inhomogeneous electric fields were used to exerted forces on the atoms prepared in superpositions of these internal states. Interference fringes, with contributions arising from the spatial separation of these Rydberg-atom wave packets in the direction of propagation of the atomic beam, were identified through changes in the internal-state populations as the magnitudes and durations of the time-dependent electric-field gradient pulses were adjusted. The maximal displacement of the atomic wave packets for which interference fringes were observed was $\sim 0.75 \mathrm{~nm}$, limited by the longitudinal velocity spread in the atomic beam and the characteristics of the inhomogeneous electric-field distribution in the apparatus. The experimental data are in good quantitative agreement with the results of numerical calculations of the time evolution of the atomic states under the experimental conditions.
\end{abstract}

DOI: 10.1103/PhysRevA.104.033305

\section{INTRODUCTION}

Rydberg states of atoms and molecules can exhibit large static electric dipole moments and large static electric dipole polarizabilities [1]. These make samples in such states particularly sensitive to electric fields [2-5] and allow forces to be exerted on them to control their motion using inhomogeneous electric fields [6,7]. This has given rise to the development of the methods of Rydberg-Stark deceleration to accelerate, decelerate, and trap cold Rydberg atoms and molecules [8,9].

For atoms or molecules prepared in coherent superpositions of Rydberg states with different static electric dipole moments or different static electric dipole polarizabilities, the methods of Rydberg-Stark deceleration can be exploited to generate coherent superpositions of momentum states for matter-wave interferometry [10,11]. This type of electric Rydberg-atom interferometry was first demonstrated with atoms in circular Rydberg states. These states have long fluorescence lifetimes, and pairs of circular states that differ in their value of the principal quantum number $n$ by one act as quasi-two-level systems with long coherence times and strong single-photon electric dipole transitions at microwave frequencies. However, the nonadiabatic evolution of these states in the fast-pulsed electric fields applied for interferometry imposed limitations on the achievable displacements of the Rydberg-atom wave packets for which interference fringes could be observed.

Here we report the results of experiments in which the limitations arising from nonadiabatic internal-state dynamics when working with circular Rydberg states have been circumvented through the use of low- $\ell$ ( $\ell$ is the orbital an- gular momentum quantum number of the Rydberg electron) states. This has allowed the observation of interference fringes between Rydberg-atom wave packets with displacements of up to $0.75 \mathrm{~nm}$, i.e., $\sim 5$ times larger than in previous experiments with circular Rydberg states. The dimensions of the atoms employed in this work, as given by the spatial extent of the Rydberg electron charge distribution, were $\sim 500 \mathrm{~nm}$, and they exhibited induced electric dipole moments of up to 9450 D. These large dimensions and electric dipole moments offer unique opportunities for tests of quantum mechanics using this approach to atom interferometry, through studies of decoherence and wave-function collapse. They are also suited to probing quantum-mechanical phases including, e.g., Kennard phases [12,13], and geometric phases associated with moving dipoles in electric and magnetic fields [14-16]. Matter-wave interferometry with atoms in high Rydberg states opens possibilities for studies of the role of coherence or decoherence in gas-phase chemical reactions at low temperatures, e.g., in resonant energy transfer reactions that occur in collisions of Rydberg atoms with polar ground-state molecules [17-19]. The use of electric Rydberg-atom interferometry for measurements of acceleration in the gravitational field of Earth is of interest in tests of the weak equivalence principle for neutral particles containing antimatter [20], in particular, those involving positronium.

Electric Rydberg-atom interferometry is an electric analog of magnetic Stern-Gerlach interferometry employed in experiments with ground-state atoms [21-23]. Recent advances in Stern-Gerlach interferometry with laser cooled rubidium atoms led to the demonstration of coherent momentum splitting and, subsequently, a complete Stern- 


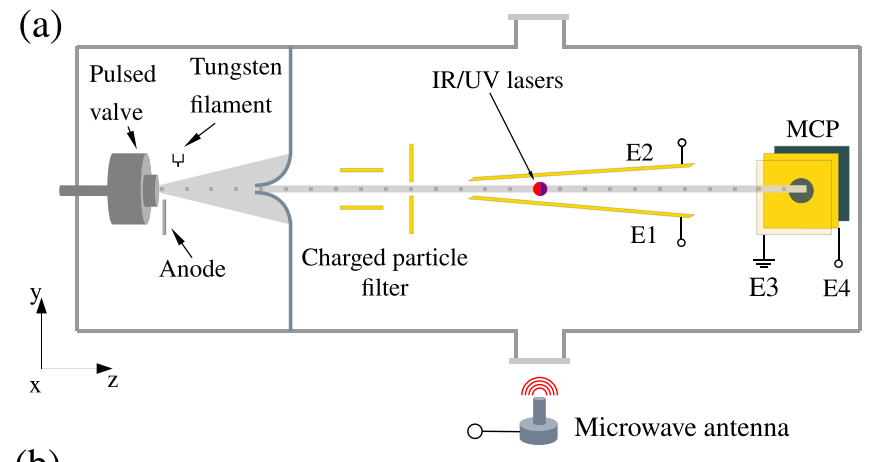

(b)
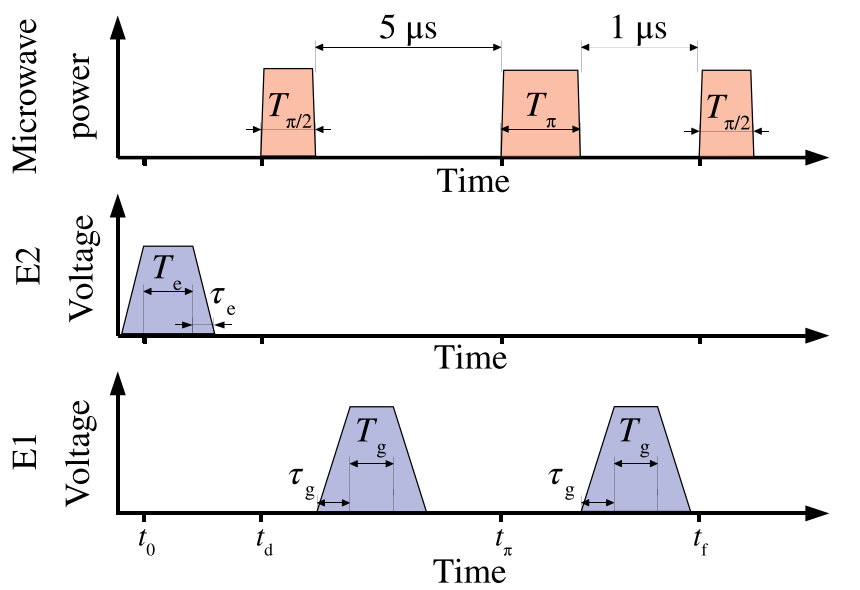

FIG. 1. (a) Schematic of the experimental apparatus. (b) Sequence of microwave and voltage pulses applied to perform electric Rydberg-atom interferometry (see the text for details).

Gerlach interferometer in the inhomogeneous magnetic fields generated close to the surfaces of atom chips $[24,25]$. And the realization of full-loop interferometers in which the phase difference $\Delta \phi$ between the interferometer arms depends strongly on the period of time $T$ over which the interferometry scheme is implemented, i.e., $\Delta \phi \propto T^{3}$ [26,27]. This strong dependence of $\Delta \phi$ on $T$ is appealing for applications in quantum sensing.

In the following, the apparatus used in the experiments is outlined in Sec. II. The numerical methods employed to calculate the time evolution of the atomic states in the interferometer are then described in Sec. III. The results of the experiments are presented and discussed in Sec. IV where they are also compared with the results of the numerical calculations performed to aid in their interpretation. Finally, in Sec. V conclusions are drawn.

\section{EXPERIMENTAL APPARATUS AND PROCEDURE}

A schematic of the experimental apparatus is shown in Fig. 1(a). A pulsed supersonic beam of helium atoms in the metastable $1 s 2 s{ }^{3} S_{1}$ level was generated in a dc electric discharge at the exit of a pulsed valve [28]. The mean velocity of the atoms in this beam was $\vec{v}_{0} \approx(0,0,2000) \mathrm{m} / \mathrm{s}$, and the pulsed valve was operated at a repetition rate of $50 \mathrm{~Hz}$. After the atoms passed through a skimmer with a 2-mm-diameter aperture, charged particles generated in the discharge were removed in an electrostatic filter. The resulting neutral-atom beam then entered the excitation and interferometry region between a pair of copper electrodes labeled E1 and E2 in Fig. 1(a). These electrodes were 70-mm wide and 105-mm long in the $x$ and $z$ dimensions, respectively, and were oriented in a wedge configuration. At the end closest to (furthest from) the skimmer they were separated in the $y$ dimension by $11.5 \mathrm{~mm}(29.7 \mathrm{~mm})$. Between E1 and E2 laser photoexcitation to the $1 s 56 s^{3} S_{1}(|56 s\rangle)$ Rydberg level was performed using the $1 s 2 s^{3} S_{1} \rightarrow 1 s 3 p{ }^{3} P_{2} \rightarrow 1 s 56 s^{3} S_{1}$ two-color two-photon excitation scheme [5]. This was driven by co-propagating $\mathrm{cw}$ laser radiation at wavelengths of $\lambda_{\mathrm{UV}}=388.975$ and $\lambda_{\mathrm{IR}}=$ $786.736 \mathrm{~nm}$ for each step, respectively.

After photoexcitation the Rydberg atoms were subjected to a $\pi / 2-\pi-\pi / 2$ Hahn-echo sequence of microwave pulses which coherently transferred population between the $|56 s\rangle$ and $1 s 57 s^{3} S_{1}(|57 s\rangle)$ levels. The $|56 s\rangle \rightarrow|57 s\rangle$ transition was driven on resonance as a single-color two-photon transition at a microwave frequency of $\omega_{56 s, 57 s} / 2=2 \pi \times$ $18.530975 \mathrm{GHz}$. In the time intervals between the microwave pulses, pulsed voltages were applied to E1 to generate inhomogenous electric fields at the position of the atoms and exert state-dependent forces on them.

The sequence of microwave and voltage pulses employed in the experiments is depicted in Fig. 1(b). At time $t_{0}$ a pulsed potential was applied to E2 to tune the transition the $|56 s\rangle$ level into resonance with the laser beams which were stabilized off-resonance form the field-free transition frequency. This pulsed potential had a rise time of $\tau_{\mathrm{e}}=370 \mathrm{~ns}$ and was maintained at its maximal amplitude for a time $T_{\mathrm{e}}$. The value of $T_{\mathrm{e}}$ determined the spatial extent of the ensemble of Rydberg atoms excited in the $z$ dimension. $T_{\mathrm{e}}$ was set to either 800 or $1800 \mathrm{~ns}$ such that the excited ensemble had a length in the $z$ dimension of $L_{\mathrm{Ry}}=v_{0} T_{\mathrm{e}} \simeq 1.6$ or $\simeq 3.6 \mathrm{~mm}$, respectively.

To configure the apparatus in Fig. 1(a) for electric Rydberg-atom interferometry, microwave spectra of the two-photon $|56 s\rangle \rightarrow|57 s\rangle$ transition were first recorded to measure and minimize the average stray electric field in the $y$ dimension between $\mathrm{E} 1$ and $\mathrm{E} 2$ on the axis of propagation of the atomic beam. This was achieved by adjusting the offset potential applied to E2, with the potential applied to E1 set to $V_{\text {grad }}=0 \mathrm{~V}$.

To implement the interferometry scheme, at the time $t_{\mathrm{d}} \simeq$ $t_{0}+2400 \mathrm{~ns}$, a first $\pi / 2$ pulse of microwave radiation was applied for $T_{\pi / 2} \simeq 150 \mathrm{~ns}$. This resulted in the preparation of an equal superposition of the $|56 s\rangle$ and $|57 s\rangle$ states. A first electric-field gradient pulse was then generated at the position of the atoms by applying a pulsed potential with amplitude $V_{\text {grad }}$ to E1. This pulse had rise and fall times of $\tau_{\mathrm{g}}=130 \mathrm{~ns}$, and a duration $T_{\mathrm{g}}$ [see Fig. 1(b)]. After $V_{\text {grad }}$ returned to $0 \mathrm{~V}$, the $\pi$ pulse in the Hahn-echo sequence was applied at the time $t_{\pi}=t_{\mathrm{d}}+T_{\pi / 2}+5000 \mathrm{~ns}$. This pulse had a duration $T_{\pi} \simeq 2 T_{\pi / 2}$ and was followed by a second electric-field gradient pulse with nominally identical values of $V_{\mathrm{grad}}, \tau_{\mathrm{g}}$, and $T_{\mathrm{g}}$ to the first. From measurements made in the apparatus with a high impedance probe, differences in the amplitudes of the two pulsed potentials on the order of $\pm 1 \%$ were observed. These amplitude differences increased with the value of $\left|V_{\text {grad }}\right|$. A final $\pi / 2$ pulse was applied $1000 \mathrm{~ns}$ after the $\pi$ pulse to complete the interferometry sequence. 
The durations of the microwave pulses required to achieve $\pi / 2$ and $\pi$ rotations of the population between the $|56 s\rangle$ and the $|57 s\rangle$ states were determined by time-domain measurements of Rabi oscillations. For a complete 8.975- $\mu$ s-long pulse sequence, extending from the time of laser photoexcitation to the final $\pi / 2$ pulse, these coherent rotations on the Bloch sphere were applied at times $t_{0}+2460, t_{0}+7620$, and $t_{0}+8840 \mathrm{~ns}$. To achieve a $\pi / 2$ rotation at the position of the atoms at the first of these times, a microwave pulse duration of $160 \mathrm{~ns}$ was required. The subsequent $\pi$ and $\pi / 2$ rotations required pulses of 210 and $135 \mathrm{~ns}$ duration, respectively. The deviation of the ratios of these pulses from those expected under ideal conditions arose because of the inhomogeneity of the microwave field between E1 and E2 [11].

After the application of the final $\pi / 2$ microwave pulse, the atoms traveled for $\sim 60 \mu$ s to the detection region in the apparatus between E3 and E4. A slowly rising pulsed potential was then applied to E3, with E4 was maintained at $0 \mathrm{~V}$ to generate a time-dependent electric field that ionized the Rydberg atoms. The resulting electrons were collected on a microchannel plate (MCP) detector. Because of the time dependence of the ionization field, the electron signal associated with the $|57 s\rangle$ state arrived at the MCP earlier than that from the $|56 s\rangle$ state [29]. Quantum-state-selective measurements of the Rydberg state populations were, therefore, made by monitoring the arrival times of the electrons at the MCP.

\section{THEORETICAL BACKGROUND}

In the approach to electric Rydberg-atom interferometry described here, quantum-state-dependent forces were exerted on atoms prepared in coherent superpositions of Rydberg states with different electric dipole polarizabilities using inhomogeneous electric fields. This resulted in the generation of coherent superpositions of momentum states. Interference fringes with contributions arising as a result of the spatial separation between these states were observed by monitoring the internal Rydberg state populations at the output of the interferometer.

\section{A. Electric Rydberg-atom interferometry with internal-state labeling}

In an electric Rydberg-atom interferometer the external motional state of an atom is coherently split into spatially separated momentum components. The difference in the phase $\Delta \phi_{\text {tot }}(t)$, accumulated between these components, i.e., between the arms of the interferometer, can, by analogy with the treatment in Refs. [30,31] for a light-pulse atom interferometer, be expressed as

$$
\Delta \phi_{\text {tot }}(t)=\Delta \phi_{\text {action }}(t)+\Delta \phi_{\text {separation }}(t),
$$

where $\Delta \phi_{\text {action }}(t)$ and $\Delta \phi_{\text {separation }}(t)$ arise from the differences in the classical action in the interferometer arms and the spatial separation of the Rydberg-atom wave packets, respectively.

The contribution to the phase arising from the action in each of two arms $i=1,2$ is

$$
\phi_{\text {action, }, i}(t)=\frac{1}{\hbar} \int_{0}^{t} \mathcal{L}_{i} d t,
$$

here $\mathcal{L}_{i}=T_{i}-V_{i}$ is the Lagrangian with kinetic-energy $T_{i}$ and potential-energy $V_{i}$, and $\hbar$ is the reduced Planck constant. In the case of interest here,

$$
\phi_{\text {action, } i}(t)=\frac{1}{\hbar}\left[\frac{m_{\mathrm{He}}}{2} \int_{0}^{t} v_{i}^{2} d t-\int_{0}^{t} W_{S, i}(t) d t\right],
$$

where $m_{\mathrm{He}}$ is the mass of the helium atom and the potentialenergy $W_{S, i}(t)$ represents the total internal energy of the atom including the Stark shift. The phase difference can, therefore, be separated into a dynamic part $\Delta \phi_{\text {dynamic }}(t)$, that depends on the difference in the kinetic energies of the wave packets in each arm of the interferometer, and a part $\Delta \phi_{\text {Stark }}(t)$ that depends on the Stark energy in each arm such that

$$
\Delta \phi_{\text {action }}(t)=\Delta \phi_{\text {dynamic }}(t)-\Delta \phi_{\text {Stark }}(t) .
$$

The separation phase difference in Eq. (1) can be expressed as $[30,31]$

$$
\Delta \phi_{\text {separation }}(t)=\frac{1}{\hbar}\left(\frac{p_{1}+p_{2}}{2}\right)\left(z_{1}-z_{2}\right),
$$

where $p_{i}=p_{0}+\Delta p_{i}$ and $z_{i}=z_{0}+\Delta z_{i}$ are the momentum and classical position, respectively, of the wave packets in an interferometer with arms spatially separated in the $z$ dimension, an initial momentum $p_{0}$ (position $z_{0}$ ), and changes in momentum $\Delta p_{i}$ (position $\Delta z_{i}$ ). Since the de Broglie wavelength $\lambda_{\mathrm{dB}}=h / p$, if the change in momentum in each arm of the interferometer is much smaller than the initial momentum $\left(p_{1}+p_{2}\right) / 2 \simeq p_{0}$ and the separation phase,

$$
\Delta \phi_{\text {separation }}(t) \simeq-\frac{2 \pi \Delta z}{\lambda_{\mathrm{dB}_{0}}} .
$$

To calculate the phase evolution of the quantum states in the interferometer implemented here a semiclassical treatment was carried out. In this treatment each Rydberg-atom wave packet was assumed to follow the classical path of least action through the interferometer, i.e., the expectation values of the position and momentum operators followed the classical equations of motion,

$$
m_{\mathrm{He}} \frac{d}{d t}\left\langle z_{i}\right\rangle=\left\langle p_{i}\right\rangle,
$$

and

$$
\frac{d}{d t}\left\langle p_{i}\right\rangle=-\nabla W_{S, i}(t) .
$$

At time $t=0$, considered to be immediately after the first $\pi / 2$ pulse in Fig. 1(b), an atom in the interferometer occupied the state,

$$
|\psi(t=0)\rangle=\frac{1}{\sqrt{2}}\left[\left|n_{1}(0)\right\rangle\left|p_{1}(0)\right\rangle+\left|n_{2}(0)\right\rangle\left|p_{2}(0)\right\rangle\right],
$$

where $\left|n_{i}(t)\right\rangle$ and $\left|p_{i}(t)\right\rangle$ represent the internal Rydberg state with principal quantum number $n$ and the external momentum state in arm $i$, respectively. When $t=0,\left|p_{1}(0)\right\rangle=\left|p_{2}(0)\right\rangle$, but for $t>0$, the gradient of the potential $W_{S, i}(t)$ caused a separation of the Rydberg-atom wave packets. After a time $t^{\prime}$, the state of the atom, therefore, evolved to

$$
\begin{aligned}
\left|\psi\left(t=t^{\prime}\right)\right\rangle= & \frac{1}{\sqrt{2}}\left[e^{-i \phi_{1}\left(t^{\prime}\right)}\left|n_{1}\left(t^{\prime}\right)\right\rangle\left|p_{1}\left(t^{\prime}\right)\right\rangle+\cdots\right. \\
& \left.e^{-i \phi_{2}\left(t^{\prime}\right)}\left|n_{2}\left(t^{\prime}\right)\right\rangle\left|p_{2}\left(t^{\prime}\right)\right\rangle\right],
\end{aligned}
$$

where $\phi_{i}$ is the phase accumulated in arm $i$. 
At time $t=t_{\pi}$ in Fig. 1(b), the internal Rydberg-state population was inverted upon the application of a $\pi$ rotation on the Bloch sphere. In the experiments this coherent internal state inversion was performed in zero electric field. In the calculations was assumed to occur instantaneously at the midpoint of the $\pi$ pulse at time $t_{\pi}+T_{\pi} / 2$ in Fig. 1(b) such that

$$
n_{1}(t)= \begin{cases}56, & 0 \leqslant t<t_{\pi}+T_{\pi} / 2, \\ 57, & t_{\pi}+T_{\pi} / 2 \leqslant t<t_{\mathrm{f}},\end{cases}
$$

and

$$
n_{2}(t)= \begin{cases}57, & 0 \leqslant t<t_{\pi}+T_{\pi} / 2 \\ 56, & t_{\pi}+T_{\pi} / 2 \leqslant t<t_{\mathrm{f}} .\end{cases}
$$

After the application of the second electric-field gradient pulse, the interferometry sequence was completed at time $t=t_{\mathrm{f}}$ with a final $\pi / 2$ rotation of the internal Rydberg-state population on the Bloch sphere. This final $\pi / 2$ pulse resulted in the generation of a superposition of each internal Rydberg state with each of the final momentum states such that

$$
\begin{aligned}
\left|\psi\left(t=t_{\mathrm{f}}\right)\right\rangle= & \frac{1}{2}\left\{e^{-i \phi_{1}\left(t_{\mathrm{f}}\right)}[|56\rangle+|57\rangle]\left|p_{1}\left(t_{\mathrm{f}}\right)\right\rangle+\cdots\right. \\
& \left.e^{-i \phi_{2}\left(t_{\mathrm{f}}\right)}[|56\rangle-|57\rangle]\left|p_{2}\left(t_{\mathrm{f}}\right)\right\rangle\right\} .
\end{aligned}
$$

Rydberg-state-selective detection, implemented in the experiments by pulsed electric-field ionization, was realized in the calculations by the application of the measurement operator $\hat{M}=|n\rangle\langle n| \otimes \mathbb{I}$, where $\mathbb{I}$ is the identity. The probability of measuring either Rydberg state was, therefore, given by the trace $\operatorname{Tr}\left[\hat{M}\left|\psi\left(t_{\mathrm{f}}\right)\right\rangle\left\langle\psi\left(t_{\mathrm{f}}\right)\right|\right]$. Thus, the population of, e.g., the $|57 s\rangle$ state $P_{57 s}$ was

$$
P_{57 s}=\frac{1}{2}\left\{1+C \cos \left[\Delta \phi_{\mathrm{tot}}\left(t_{\mathrm{f}}\right)\right]\right\},
$$

where $\Delta \phi_{\text {tot }}\left(t_{\mathrm{f}}\right)=\phi_{2}\left(t_{\mathrm{f}}\right)-\phi_{1}\left(t_{\mathrm{f}}\right)$ as given by Eq. (1), and the contrast $C$ reflected the spatial overlap of the wave packets in each arm of the interferometer at time $t_{\mathrm{f}}$, i.e., $\left\langle p_{1}\left(t_{\mathrm{f}}\right) \mid p_{2}\left(t_{\mathrm{f}}\right)\right\rangle$.

In this semiclassical approach to calculating Rydberg-atom matter-wave interference patterns, the finite coherence length of the Rydberg-atom wave packets was not treated explicitly, i.e., for all cases considered $C=1$ in Eq. (12). To account for the effect of the coherence length in the experiments, calculations were performed for ensembles of atoms sampled randomly from initial phase-space distributions reflecting those of the excited atoms in the experiments. More complete treatments of this type of interferometry in which effects arising from the coherence length of the atomic wave packets are considered explicitly can, however, be found in the literature, for example, in Ref. [26].

\section{B. The Stark effect in $1 s n s{ }^{3} S_{1}$ Rydberg states in helium}

The $1 s 56 s^{3} S_{1}(|56 s\rangle)$ and $1 s 57 s^{3} S_{1}(|57 s\rangle)$ Rydberg states in helium have fluorescence lifetimes in excess of $130 \mu \mathrm{s}$ and static electric dipole polarizabilities of 2.3 and $2.6 \mathrm{GHz} \mathrm{V}^{-1} \mathrm{~cm}^{-2}$, respectively. These electric dipole polarizabilities and, more generally, the complete Stark shifts of these levels which were used in all interferometry calculations, were obtained from the eigenvalues of the Hamiltonian matrix for the atom in the presence of an electric field. This Hamiltonian was constructed in a $\left|n \ell m_{\ell}\right\rangle$ basis, where $m_{\ell}$ is the azimuthal quantum number of the Rydberg electron.
TABLE I. Quantum defects $\delta_{n \ell}$ of the triplet Rydberg states in helium with $n=56$ and 57 [36].

\begin{tabular}{lcc}
\hline \hline$\ell$ & $\delta_{56 \ell}$ & $\delta_{57 \ell}$ \\
\hline 0 & 0.2966688 & 0.2966684 \\
1 & 0.0683543 & 0.0683545 \\
2 & 0.0028893 & 0.0028894 \\
3 & 0.0004468 & 0.0004468 \\
4 & 0.0001269 & 0.0001269 \\
5 & 0.0000486 & 0.0000486 \\
\hline \hline
\end{tabular}

The diagonal elements of this Hamiltonian were determined using the Rydberg formula with nonzero quantum defects for the triplet states with $\ell \leqslant 6$ as given in Table I. The off-diagonal matrix elements associated with the interaction with the electric field contained angular integrals that were calculated using standard angular momentum algebra [32] and radial integrals that were calculated using the Numerov method with a pure $-1 / r$ potential [33]. In the triplet Rydberg states in helium with $n=56$ and 57, the largest spin-orbit splittings, i.e., those of the ${ }^{3} P_{J}$ levels, are less than $1.3 \mathrm{MHz}$ [34] and do not contribute significantly to the Stark shifts of the $|56 s\rangle$ and $|57 s\rangle$ states over the range of electric fields employed in the experiments. This approach to calculating the Stark energy-level structure in helium agrees with experimental measurements to a precision of $\sim 30 \mathrm{MHz}$ for electric fields up to the classical ionization field [35].

In the presence of an inhomogenous electric field with a gradient in the $z$ dimension, i.e., along the axis of propagation of the atomic beam in Fig. 1(a), the Rydberg-state-dependent force exerted on an atom is given by

$$
f_{z}=-\nabla W_{S}(t, z),
$$

where $W_{S}(t, z)$ is the Stark energy which depends on the position in the $z$ dimension. In the one-dimensional model considered in the calculations, the electric-field $F(z, t)$ within the wedge-shaped electrode structure on the axis of propagation of the atomic beam had the form

$$
F(z, t)=\frac{V(t)}{s(z)}+\mathcal{F}(z),
$$

where $V(t)$ is the potential difference between E1 and E2, $s(z)$ is the $z$-position-dependent separation between E1 and $\mathrm{E} 2$ in the $y$ dimension, and $\mathcal{F}(z)=\left(z-z_{\text {cancel }}\right)\left(d \mathcal{F}_{\text {stray }} / d z\right)$ represents a residual position-dependent stray electric field. This residual stray electric field was canceled at the position $z_{\text {cancel }}$ and had a gradient $d \mathcal{F}_{\text {stray }} / d z$.

To aid in the interpretation of the experimental data, classical trajectories of atoms in the inhomogeneous electric field described by Eq. (14) were calculated numerically with an integration time-step size of $0.25 \mathrm{~ns}$. In these calculations the trajectories of 200 atoms through the interferometer were determined. The initial longitudinal velocity of each was generated by random sampling from a Gaussian distribution with mean of $v_{0}=2000 \mathrm{~m} / \mathrm{s}$ and a standard deviation of $50 \mathrm{~m} / \mathrm{s}$ as determined by time-of-flight measurements [37]. Because the propagation distance of the atoms in the time $T_{\mathrm{e}}$, when the excitation electric-field pulse was applied to E2 [see Fig. 1(b)] was large, i.e., $\left(L_{\mathrm{Ry}}=v_{0} T_{\mathrm{e}}>1 \mathrm{~mm}\right)$ compared to the waist 


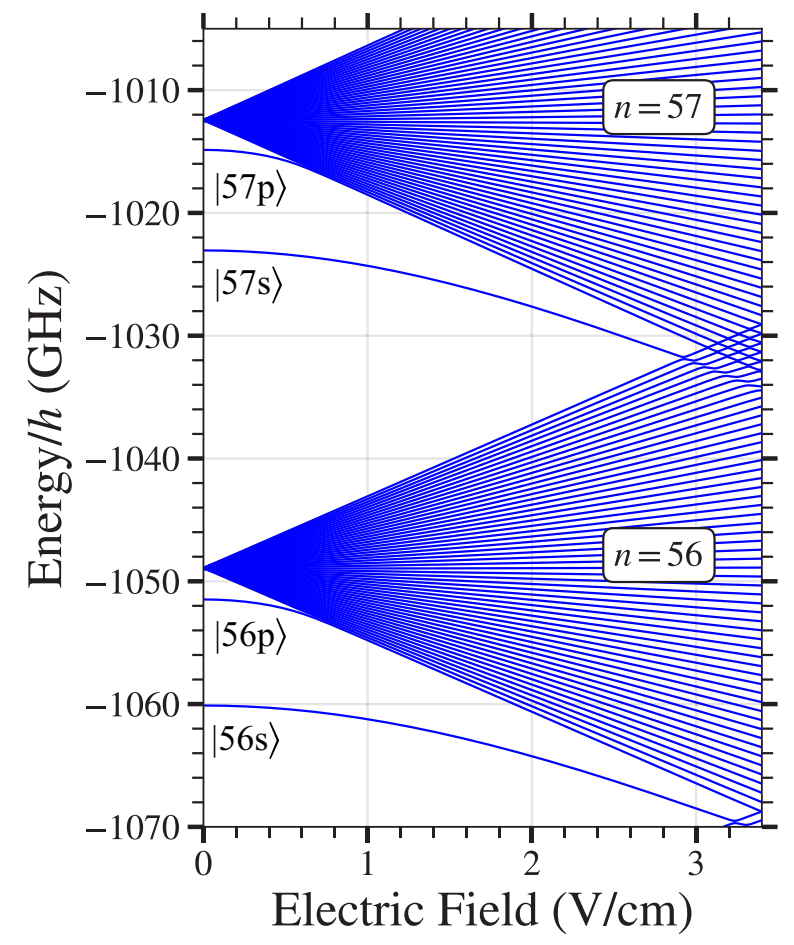

FIG. 2. Energy-level structure of the triplet Rydberg states in helium with $n=56$ and 57 .

of the focused laser beams used for Rydberg-state photoexcitation $[\sim 100 \mu \mathrm{m}$ full width at half maximum (FWHM)], the initial longitudinal position of each atom at the time $t=t_{\mathrm{d}}$ of the first $\pi / 2$ microwave pulse of the interferometry sequence, was generated by random sampling from a uniform distribution of excitation times between $t=0$ and $t=T_{\mathrm{e}}$, and then calculating the distance traveled from the time of excitation to $t_{\mathrm{d}}$. At each time step in the calculations the electric-field strength and electric-field gradient at the position of each atom were determined and used, together with the calculated Stark shifts of the atomic energy levels, to determine the force acting in each arm in the interferometer. For the $|56 s\rangle$ and $|57 s\rangle$ Rydberg states, these forces resulted in accelerations of $\sim 1000 \mathrm{~m} / \mathrm{s}^{2}$ for electric-field gradients of $\sim 0.1 \mathrm{~V} / \mathrm{cm}^{2}$.

\section{RESULTS}

With the interferometer apparatus and pulse sequence optimized as described in Sec. II, pairs of electric-field gradient pulses with equal rise and fall times of $\tau_{\mathrm{g}}=130 \mathrm{~ns}$, and equal durations of $T_{\mathrm{g}}=72 \mathrm{~ns}$ were generated by applying pulsed potentials to E1 [see Fig. 1(a)]. To record Rydberg-atom matter-wave interference patterns the amplitudes $V_{\text {grad }}$ of these pulses were adjusted, and the population of the $|57 s\rangle$ state at the end of the interferometry sequence was monitored. The results of such a measurement are presented as the continuous blue curve in Fig. 3(a). The interference fringes seen in this figure reflect the change in the total phase difference between the interferometer arms $\Delta \phi_{\text {tot }}\left(t_{\mathrm{f}}\right)$ as $V_{\text {grad }}$ was adjusted. The reduction in the period of the fringes as $\left|V_{\text {grad }}\right|$ increases is a consequence of the quadratic Stark shifts of the $|56 s\rangle$ and $|57 s\rangle$ states. For all values of $V_{\text {grad }}$ in this figure the electric field experienced by the atoms remained below the InglisTeller field in which the $|n s\rangle$ states first cross the manifolds

\section{(a)}
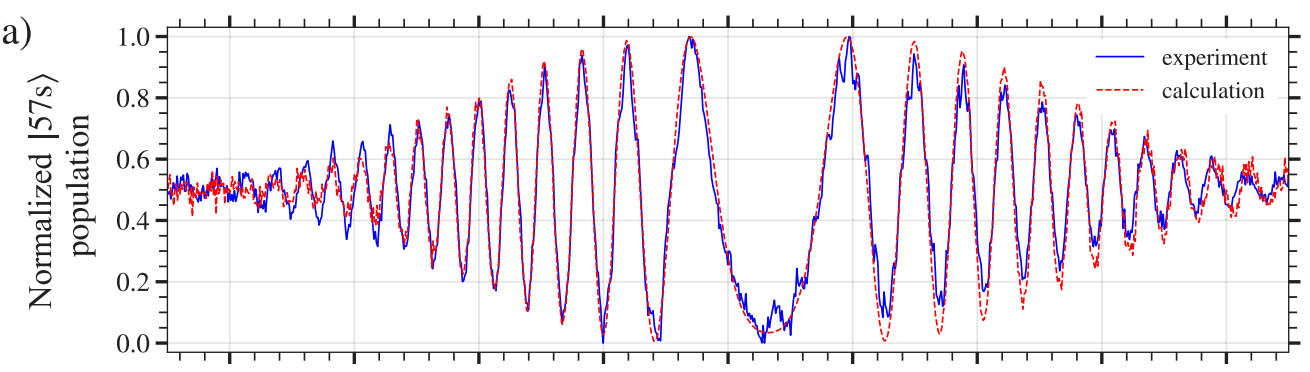

(b)

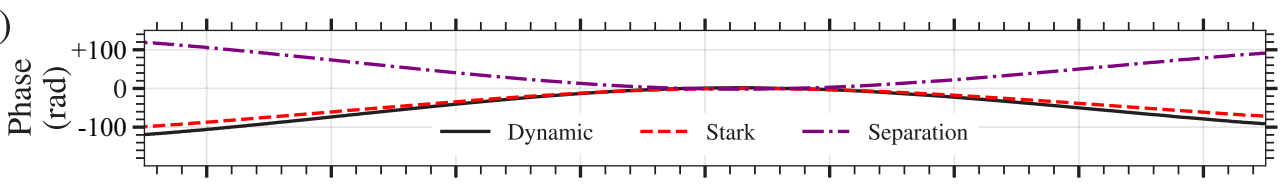

(c)

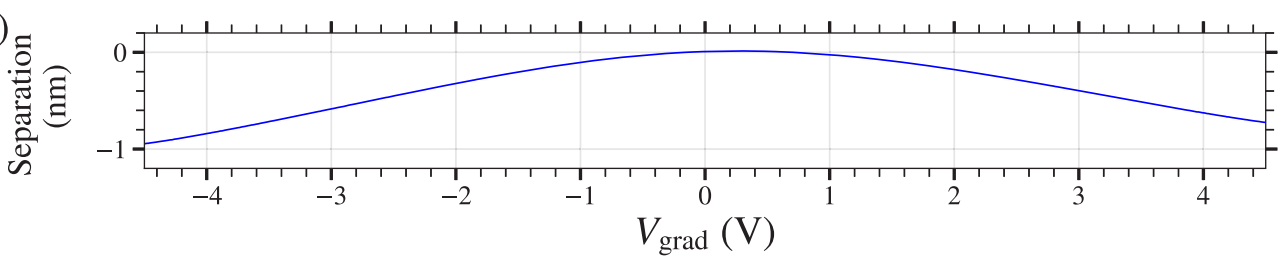

FIG. 3. (a) Measured (continuous curve) and calculated (dashed curve) Rydberg-atom interference pattern for $T_{\mathrm{g}}=72 \mathrm{~ns}$ and $\tau_{\mathrm{g}}=130 \mathrm{~ns}$. The parameter $V_{\text {grad }}$ indicated on the horizontal axis represents the amplitude of the pulsed potentials applied to electrode E1 to generate the pulsed electric-field gradients in the interferometer (see the text for details). (b) The calculated dynamic $\Delta \phi_{\text {dynamic }}\left(t_{\mathrm{f}}\right)$ (continuous black curve), Stark $\Delta \phi_{\text {Stark }}\left(t_{\mathrm{f}}\right)$ (dashed red curve), and separation $\Delta \phi_{\text {separation }}\left(t_{\mathrm{f}}\right)$ (dashed-dot violet curve) phase differences at the interferometer output that contribute to the interference pattern in (a). (c) The corresponding calculated spatial separation between the classical paths of least action at the interferometer output. 
of $\ell$-mixed hydrogenic Stark states in Fig. 2 . Up to $\sim 15$ interference minima are observed for values of $V_{\text {grad }}<0$.

In previous electric Rydberg-atom interferometry experiments performed with atoms in circular Rydberg states, the maximal displacement of the atomic wave packets for which interference fringes were observed was limited by nonadiabatic internal-state dynamics in the pulsed electric fields applied [10]. These nonadiabatic dynamics led to a loss of population, and, hence, a loss in signal from the quasi-twolevel system composed of the pair of circular states as the value of $\left|V_{\text {grad }}\right|$ was increased. It is evident from the convergence of the $|57 s\rangle$ population in Fig. 3(a) toward 0.5 for values of $\left|V_{\text {grad }}\right|$ close to $4.5 \mathrm{~V}$ that these nonadiabatic losses do not occur in the present experiments. This is because the $|n s\rangle$ Rydberg states remain energetically isolated from all other higher- $\ell$ Rydberg states over the full range of electric fields experienced by the atoms during the interferometry sequence. Nevertheless, at high values of $\left|V_{\text {grad }}\right|$ the contrast of the interference fringes does reduce.

A more detailed interpretation of the interferogram in Fig. 3(a) was obtained by comparison of the experimental data with the results of calculations (dashed red curve) performed using the methods described in Sec. III. The good quantitative agreement between the results of the experiments and the calculations allowed the origin of the reduction in the contrast, or decoherence, of the interference patterns at high values of $\left|V_{\text {grad }}\right|$ to be inferred. This decoherence directly reflects the phase-space properties of the ensemble of Rydberg atoms in the experiments, i.e., the longitudinal velocity spread and the spatial extent in the $z$ dimension.

To achieve good agreement between the results of the calculations and the experimental data in Fig. 3(a), the ratio of the amplitude of the voltage pulse applied to generate the second electric-field gradient pulse, to that applied to generate the first gradient pulse was set to 1.01 - within the range measured in the apparatus (see Sec. II). To account for the offset of the centroid of the interferogram to $V_{\text {grad }} \simeq+0.4 \mathrm{~V}$, the residual stray electric-field $\mathcal{F}(z)$ in Eq. (14) was required to have a nonzero gradient. In the experimental apparatus this residual electric-field gradient arose because although the average stray electric field experienced by the atoms during the interferometry sequence was minimized (see Sec. II), the minimization of this average field is not commensurate with the minimization of the field gradient to which the Rydberg-atom interferometer is sensitive. The calculated interference pattern in Fig. 3(a) was obtained for a stray electric-field gradient of $d \mathcal{F}_{\text {stray }} / d z=-22.5 \mathrm{mV} / \mathrm{cm}^{2}$. This stray field gradient is the cause of the more rapid phase accumulation for $V_{\text {grad }}<0$ than for $V_{\text {grad }}>0$ in the experimental data.

The individual contributions from the dynamic, Stark, and separation phase differences to $\Delta \phi_{\text {tot }}\left(t_{\mathrm{f}}\right)$ in the calculated interferogram in Fig. 3(a) are displayed in Fig. 3(b). Because of the high speeds of the atoms in the $z$ dimension in which the forces were exerted, the initial momentum $p_{0}$ was much greater than the changes in momentum resulting from the forces, i.e., $p_{0} \gg \Delta p_{i}$. Consequently, the difference in the dynamic phase in each arm of the interferometer was of approximately equal magnitude but opposite sign to the difference in the separation phase, i.e., $\Delta \phi_{\text {dynamic }} \simeq-\Delta \phi_{\text {separation. }}$ The results of the calculations in Fig. 3(b) demonstrate that under the conditions in which the experiments were performed, the Stark phase difference plays a significant role. This is because as the atoms move in the positive $z$ dimension between E1 and E2, the magnitude of the electric field they experience for each value of $V_{\text {grad }}$ reduces. As a result, the electric field at the position of the atoms, and therefore the Stark shift at the time of the first electric-field gradient pulse is slightly larger than that at their position at the time of the second gradient pulse. The Hahn-echo sequence of microwave pulses, therefore, does not completely cancel the phase accumulation associated with the Stark shifts of the Rydberg states.

The calculations also yielded information on the spatial separation between the classical paths at the interferometer output as displayed in Fig. 3(c). Comparing the interferogram in Fig. 3(a) with the results of these calculations indicates that the maximal displacement of the Rydberg-atom wave packets for which interference fringes were observed was $\sim 0.75 \mathrm{~nm}$ and, hence, $\sim 15 \lambda_{\mathrm{dB}}$. The asymmetry of the curve in Fig. 3(c) about $V_{\text {grad }}=0 \mathrm{~V}$ is a consequence of the residual stray electric-field gradient in the apparatus.

An alternative determination of the stray electric-field gradient in the interferometer was made by microwave spectroscopy of Rydberg-Rydberg transitions, performed at selected times after laser photoexcitation, and therefore longitudinal positions between E1 and E2. These spectra of the two-photon $|56 s\rangle \rightarrow|57 s\rangle$ transition were recorded using single pulses of microwave radiation with durations of $2 \mu \mathrm{s}$ and are displayed in Fig. 4. The absolute transition frequencies in these measurements are affected by Doppler shifts on the order of $+100 \mathrm{kHz}$ in the fast-moving pulsed supersonic beam. However, across this set of spectra, the measured two-photon transition frequency shifts by $\sim+60 \mathrm{kHz}$. Since the $|56 s\rangle \rightarrow$ $|57 s\rangle$ transition exhibits a quadratic Stark shift toward lower frequency with increasing electric-field strength, the observed shift toward higher transition frequencies at later times after laser photoexcitation indicates that the uncompensated stray electric field between E1 and E2 reduces toward the end of these electrodes closest to the detection region. At this end, the separation between these electrodes in the $y$ dimension is greatest, and the atoms are, therefore, furthest from the electrode surfaces and the source of residual stray fields $[38,39]$. Comparison of the experimentally measured transition frequencies in Fig. 4 with the calculated Stark shifts of the $|56 s\rangle$ and $|57 s\rangle$ states, yielded a mean residual uncompensated stray field gradient between E1 and E2 on the axis of propagation of the atomic beam of $d \mathcal{F}_{\text {stray }} / d z \simeq-20 \mathrm{mV} / \mathrm{cm}^{2}$. This is consistent with the residual gradient of $-22.5 \mathrm{mV} / \mathrm{cm}^{2} \mathrm{ob}-$ tained by comparing the measured and calculated interference patterns in Fig. 3.

The effect of the spatial extent of the ensemble of Rydberg atoms in the $z$ dimension on the coherence length in the experiments was investigated by adjusting the value of $T_{\mathrm{e}}$, the laser photoexcitation pulse duration. An interferogram recorded for $T_{\mathrm{e}}=800 \mathrm{~ns}$, i.e., with $L_{\mathrm{Ry}} \simeq 1.6 \mathrm{~mm}$, and values of $V_{\text {grad }} \geqslant 0$ is displayed in Fig. 5(a). These data were recorded with the same set of experimental parameters as those in Fig. 3(a) and exhibit clearly visible interference fringes over the full range of values of $V_{\text {grad }}$ encompassed in the figure. The data presented in Fig. 5(b) were recorded with a longer photoexcitation pulse of $T_{\mathrm{e}}=1800 \mathrm{~ns}$, i.e., with $L_{\mathrm{Ry}} \simeq 3.6 \mathrm{~mm}$. From 


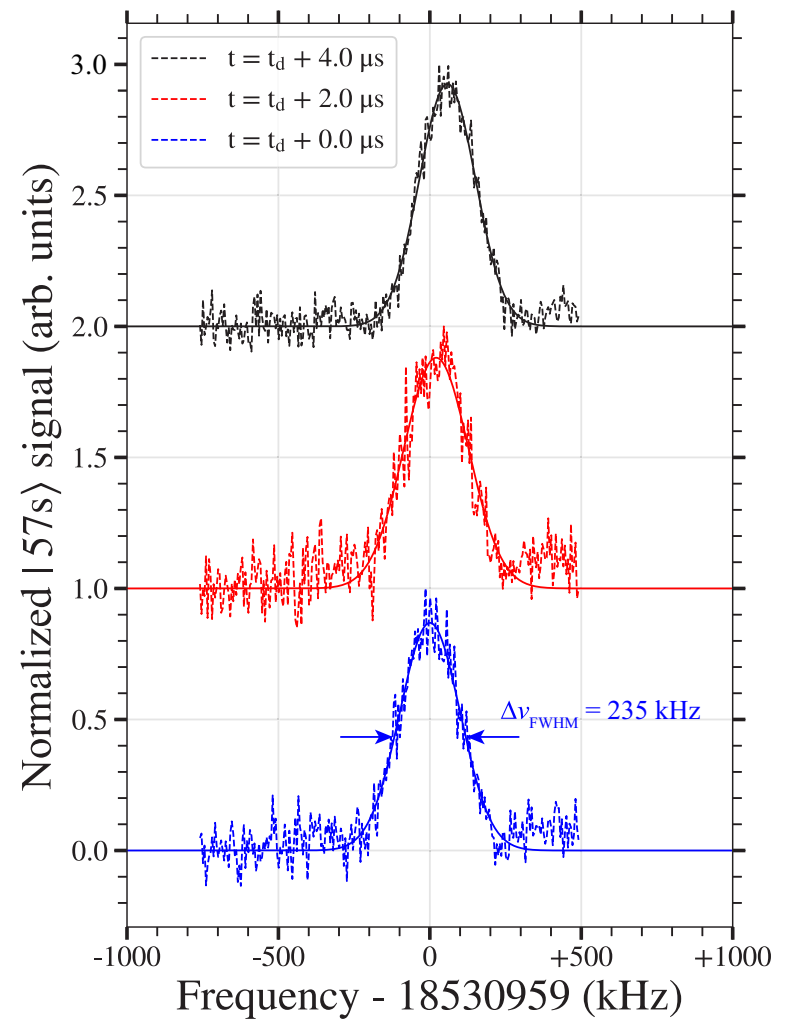

FIG. 4. Microwave spectra of the two-photon $|56 s\rangle \rightarrow|57 s\rangle$ transition recorded at times $t_{\mathrm{d}}, t_{\mathrm{d}}+2.0$, and $t_{\mathrm{d}}+4.0 \mu \mathrm{s}$ after laser photoexcitation to probe residual uncompensated stray electric-field gradients over a distance of $\sim 8 \mathrm{~mm}$ along the axis of propagation of the atomic beam between E1 and E2.

this measurement, performed under experimental conditions that were otherwise identical to those used when recording the data in panel (a), it is seen that for the longer bunch of atoms the contrast of the interference fringes reduces more rapidly as the value of $V_{\text {grad }}$ increases, indicating a reduction in the longitudinal coherence length, and rapid fluctuations are seen in the population of the $|57 s\rangle$ state. The reduction in the contrast of the interference fringes is also apparent in the results of the calculations. This is a consequence of the dependence of the electric field between E1 and E2 on the position in the $z$ dimension and the effect this has on the Stark and dynamic phase differences within the ensemble of excited atoms. The inhomogeneity of the electric field leads to different Stark shifts of, and forces on, atoms at the front and back of the excited ensemble. For a larger spatial distribution of atoms these differences are greater. Thus, the range of values of $\Delta \phi_{\mathrm{tot}}\left(t_{\mathrm{f}}\right)$ accumulated across the ensemble is also greater, and increased decoherence ensues. The rapid fluctuations, with a periodicity of $\sim 0.1 \mathrm{~V}$, seen throughout the interference pattern in Fig. 5(b) are attributed to the spatial inhomogeneity of the microwave field and the effect this has on the fidelity of the microwave pulses across the ensemble of Rydberg atoms. Because of the inhomogeneity of this field in the $z$ dimension, ideal $\pi / 2$ and $\pi$ pulses can only be achieved for compact ensembles of excited atoms that are well localized at the time of the application of each pulse. By increasing the spatial extent of the ensemble of Rydberg atoms, the set of

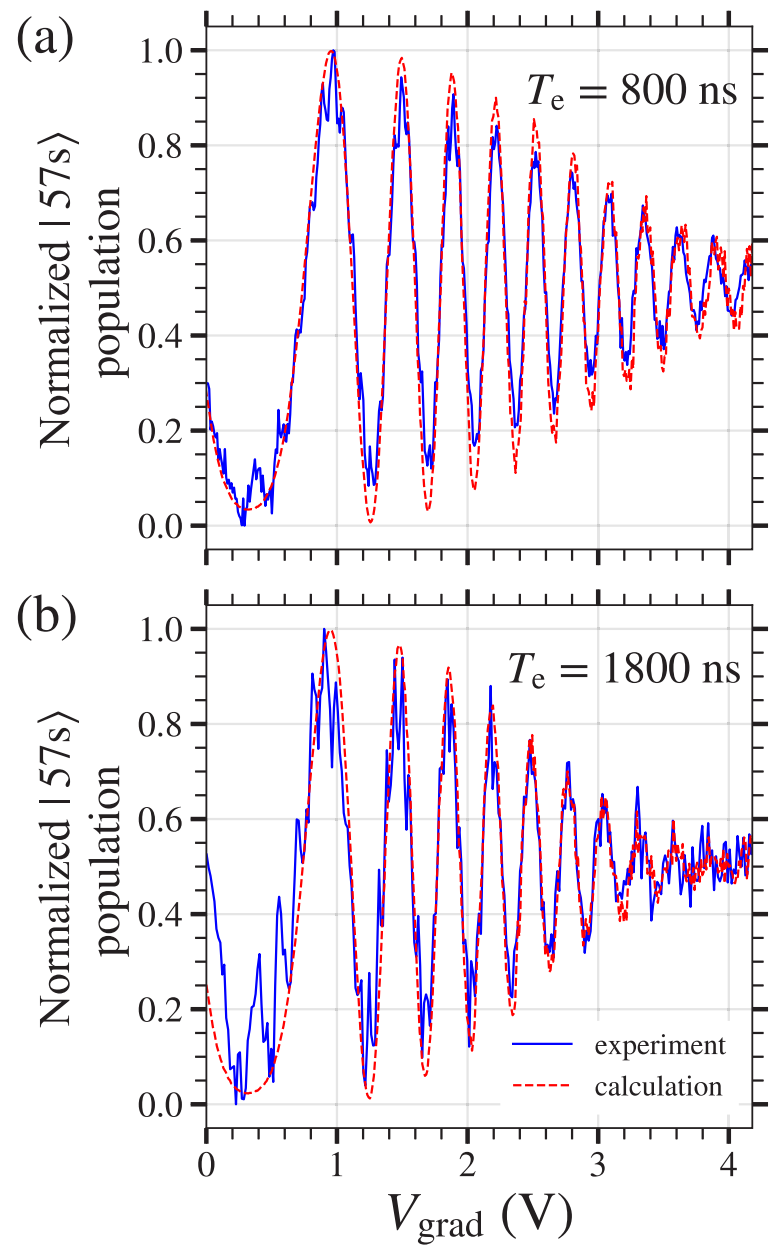

FIG. 5. Measured (continuous blue curves) and calculated (dashed red curves) Rydberg atom interference patterns recorded for $T_{\mathrm{g}}=72 \mathrm{~ns}, \tau_{\mathrm{g}}=130 \mathrm{~ns}$, and values of $V_{\text {grad }} \geqslant 0$ after laser photoexcitation of (a) a short $L_{\mathrm{Ry}} \simeq 1.6 \mathrm{~mm}$ ensemble of Rydberg atoms when $T_{\mathrm{e}}=800 \mathrm{~ns}$, and (b) a long $L_{\mathrm{Ry}} \simeq 3.6 \mathrm{~mm}$ ensemble of Rydberg atoms when $T_{\mathrm{e}}=1800 \mathrm{~ns}$.

microwave pulses used no longer yielded ideal $\pi / 2$ and $\pi$ rotations on the Bloch sphere for every atom. This resulted in a beating effect in the final Rydberg-state population which is superimposed on the main interference fringes.

Further information on decoherence in the interferometer was obtained by performing experiments with electric-field gradient pulses of a constant amplitude but durations that were adjusted. These measurements, the results of which are presented in Fig. 6, provided insight into the effects of the increased exposure time of the atoms to the electric fields in the interferometer. For these measurements $T_{\mathrm{e}}$ was set to $800 \mathrm{~ns}$, and data recorded for $V_{\text {grad }}=2$ and $1 \mathrm{~V}$ are displayed in Figs. 6(a) and 6(b), respectively (continuous curves). For the higher value of $V_{\text {grad }}=2 \mathrm{~V}$ in Fig. 6(a), the differences in the induced electric dipole moments and therefore the differences in the state-dependent forces exerted in each arm in the interferometer were greatest. As a result, $\Delta \phi_{\mathrm{tot}}\left(t_{\mathrm{f}}\right)$ accumulated rapidly as the duration of the gradient pulses increased. The dependence of the contrast of the measured interference fringes in Fig. 6(a) on the value of $T_{\mathrm{g}}$ is similar to 

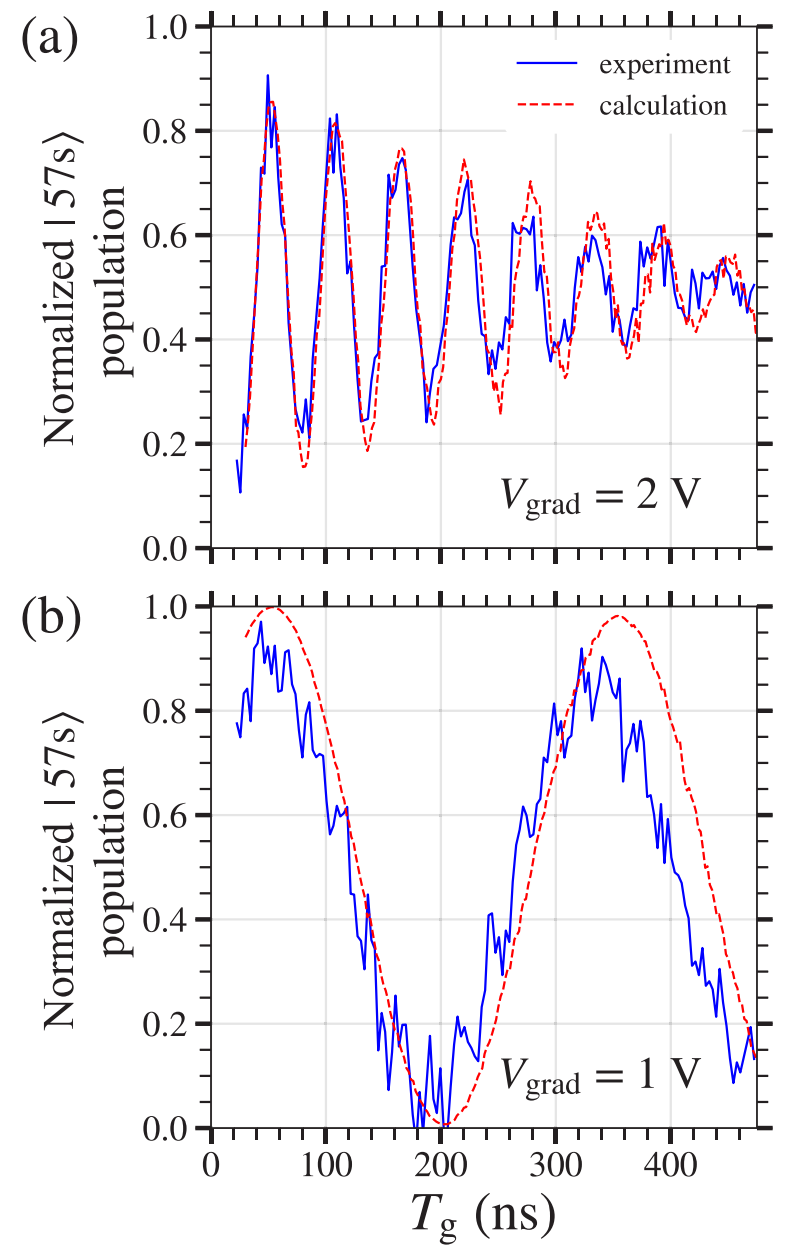

FIG. 6. Measured (continuous blue curves) and calculated (dashed red curves) Rydberg-atom interference patterns recorded by adjusting the electric-field gradient pulse duration $T_{\mathrm{g}}$ for $\tau_{\mathrm{g}}=130 \mathrm{~ns}$, and (a) $V_{\text {grad }}=2 \mathrm{~V}$, and (b) $V_{\text {grad }}=1 \mathrm{~V}$. In each case $T_{\mathrm{e}}=800 \mathrm{~ns}$.

that observed in the calculations. This is also the case in the data recorded for $V_{\text {grad }}=1 \mathrm{~V}$. Again these data are in good quantitative agreement with the results of the calculations with the only parameter adjusted being the value of $V_{\text {grad }}$. The calculations performed do not account for decoherence that depends on the value of $V_{\text {grad }}$, i.e., decoherence arising from electric-field noise to which the more strongly polarized atoms in Fig. 6(a) when $V_{\text {grad }}=2 \mathrm{~V}$ may be more sensitive than those, e.g., in Fig. 6(b). This suggests that decoherence caused by laboratory electric-field noise does not significantly impact the performance of the interferometer over the timescales, and for the fields and field gradients used in these experiments. These data further support the conclusion that the decoherence, or coherence length, in the experiments is dominated by the combination of the longitudinal velocity spread in the ensemble of excited atoms and the dependence of the electricfield distribution within the interferometer on the longitudinal position.

\section{CONCLUSION}

The use of low- $\ell$ Rydberg states in the electric Rydbergatom interferometry experiments described here has allowed limitations on the maximal achievable displacement of the atomic wave packets, arising as a result of nonadiabatic internal-state dynamics, encountered previously with atoms in circular Rydberg states to be overcome. This has meant that effects of interference between Rydberg-atom wave packets separated by up to $\sim 0.75 \mathrm{~nm}$ were observable.

To generate superpositions of Rydberg-atom wave packets with larger spatial separations, it will be necessary to overcome limitations arising from the velocity spread in the atomic beam and the position dependence of the electric-field gradient within the wedge configuration of electrodes used in the experiments. This may be achieved by: (1) operating the interferometer in a transverse geometry to take advantage of the lower transverse velocity spread $(\sim \pm 1 \mathrm{~m} / \mathrm{s})$ in the collimated supersonic beam, (2) exploiting sequences of shaped electric-field gradient pulses, and (3) devising more elaborate electrode geometries to optimize the spatial distribution of the inhomogeneous electric fields in the interferometer. These developments will require a detailed understanding of, and enhanced control over contributions from the individual dynamic, Stark, and separation phases to the total phase difference between the interferometer arms. For atoms moving at high speeds and, therefore, with short de Broglie wavelengths as in the experiments reported here, when the spatial separation between the interferometer arms is small the separation and dynamic phase differences cancel. However, this behavior does not persist at low speeds and for larger separations. Under such conditions the Rydberg-atom interferograms are expected to be significantly more sensitive to the details of the time and position dependence of the electric fields. By shaping the pulsed potentials applied to generate the electricfield gradients in the time domain, it is anticipated that the relative contributions from the dynamic, Stark, and separation phases to the measured interferograms can be controlled.

The advances in coherent Rydberg-atom optics demonstrated here open opportunities in a range of research areas. These include applications in quantum sensing and investigations of geometric phases for particles with large electric dipole moments; studies of the role of coherence or decoherence in gas-phase chemical reactions at low temperatures; and tests of the weak equivalence principle with Rydberg positronium atoms.

\section{ACKNOWLEDGMENTS}

This work was supported by the European Research Council (ERC) under the European Union's Horizon 2020 Research and Innovation Program (Grant No. 683341), and the Engineering and Physical Sciences Research Council through the EPSRC Centre for Doctoral Training in Delivering Quantum Technologies.
[1] T. F. Gallagher, Rydberg Atoms (Cambridge University Press, Cambridge, UK, 1994).
[2] M. T. Frey, X. Ling, B. G. Lindsay, K. A. Smith, and F. B. Dunning, Use of the Stark effect to minimize residual electric 
fields in an experimental volume, Rev. Sci. Instrum. 64, 3649 (1993).

[3] A. Schramm, J. M. Weber, J. Kreil, D. Klar, M.-W. Ruf, and H. Hotop, Laser Photoelectron Attachment to Molecules in a Skimmed Supersonic Beam: Diagnostics of Weak Electric Fields and Attachment Cross Sections Down to $20 \mu \mathrm{eV}$, Phys. Rev. Lett. 81, 778 (1998).

[4] A. Osterwalder and F. Merkt, Using High Rydberg States as Electric Field Sensors, Phys. Rev. Lett. 82, 1831 (1999).

[5] S. D. Hogan, Y. Houston, and B. Wei, Laser photoexcitation of Rydberg states in helium with $n>400$, J. Phys. B: At., Mol. Opt. Phys. 51, 145002 (2018).

[6] W. H. Wing, Electrostatic Trapping of Neutral Atomic Particles, Phys. Rev. Lett. 45, 631 (1980).

[7] T. Breeden and H. Metcalf, Stark Acceleration of Rydberg Atoms in Inhomogeneous Electric Fields, Phys. Rev. Lett. 47, 1726 (1981).

[8] E. Vliegen, H. J. Wörner, T. P. Softley, and F. Merkt, Nonhydrogenic Effects in the Deceleration of Rydberg Atoms in Inhomogeneous Electric Fields, Phys. Rev. Lett. 92, 033005 (2004).

[9] S. D. Hogan, Rydberg-Stark deceleration of atoms and molecules, EPJ Tech. Instrum. 3, 2 (2016).

[10] J. E. Palmer and S. D. Hogan, Electric Rydberg-Atom Interferometry, Phys. Rev. Lett. 122, 250404 (2019).

[11] J. E. Palmer and S. D. Hogan, Matter-wave interferometry with atoms in high Rydberg states, Mol. Phys. 117, 3108 (2019).

[12] E. H. Kennard, Zur Quantenmechanik einfacher Bewegungstypen, Z. Phys. 44, 326 (1927).

[13] E. H. Kennard, The quantum mechanics of an electron or other particle, J. Franklin Inst. 207, 47 (1929).

[14] X.-G. He and B. H. J. McKellar, Topological phase due to electric dipole moment and magnetic monopole interaction, Phys. Rev. A 47, 3424 (1993).

[15] M. Wilkens, Quantum Phase of a Moving Dipole, Phys. Rev. Lett. 72, 5 (1994).

[16] S. Lepoutre, A. Gauguet, G. Trénec, M. Büchner, and J. Vigué, He-McKellar-Wilkens Topological Phase in Atom Interferometry, Phys. Rev. Lett. 109, 120404 (2012).

[17] V. Zhelyazkova and S. D. Hogan, Electrically tuned Förster resonances in collisions of $\mathrm{NH}_{3}$ with Rydberg He atoms, Phys. Rev. A 95, 042710 (2017).

[18] F. Jarisch and M. Zeppenfeld, State resolved investigation of Förster resonant energy transfer in collisions between polar molecules and Rydberg atoms, New J. Phys. 20, 113044 (2018).

[19] K. Gawlas and S. D. Hogan, Rydberg-state-resolved resonant energy transfer in cold electric-field-controlled intrabeam collisions of $\mathrm{NH}_{3}$ with Rydberg He atoms, J. Phys. Chem. Lett. 11, 83 (2020).

[20] D. B. Cassidy and S. D. Hogan, Atom control and gravity measurements using Rydberg positronium, Int. J. Mod. Phys.: Conf. Ser. 30, 1460259 (2014).

[21] C. Miniatura, F. Perales, G. Vassilev, J. Reinhardt, J. Robert, and J. Baudon, A longitudinal Stern-Gerlach interferometer: The "beaded" atom, J. Phys. II France 1, 425 (1991).

[22] C. Miniatura, J. Robert, O. Gorceix, V. Lorent, S. Le Boiteux, J. Reinhardt, and J. Baudon, Atomic Interferences and the Topological Phase, Phys. Rev. Lett. 69, 261 (1992).
[23] S. Nic Chormaic, V. Wiedemann, C. Miniatura, J. Robert, S. Le Boiteux, V. Lorent, O. Gorceix, S. Feron, J. Reinhardt, and J. Baudon, Longitudinal Stern-Gerlach atomic interferometry using velocity selected atomic beams, J. Phys. B: At., Mol. Opt. Phys. 26, 1271 (1993).

[24] S. Machluf, Y. Japha, and R. Folman, Coherent Stern- Gerlach momentum splitting on an atom chip, Nat. Commun. 4, 2424 (2013).

[25] Y. Margalit, O. Dobkowski, Z. Zhou, O. Amit, Y. Japha, S. Moukouri, D. Rohrlich, A. Mazumdar, S. Bose, C. Henkel, and R. Folman, Realization of a complete Stern-Gerlach interferometer: Toward a test of quantum gravity, Sci. Adv. 7, eabg2879 (2021).

[26] M. Zimmermann, M. A. Efremov, A. Roura, W. P. Schleich, S. A. DeSavage, J. P. Davis, A. Srinivasan, F. A. Narducci, S. A. Werner, and E. M. Rasel, $T^{3}$-interferometer for atoms, Appl. Phys. B: Lasers Opt. 123, 102 (2017).

[27] O. Amit, Y. Margalit, O. Dobkowski, Z. Zhou, Y. Japha, M. Zimmermann, M. A. Efremov, F. A. Narducci, E. M. Rasel, W. P. Schleich, and R. Folman, $T^{3}$ Stern-Gerlach Matter-Wave Interferometer, Phys. Rev. Lett. 123, 083601 (2019).

[28] T. Halfmann, J. Koensgen, and K. Bergmann, A source for a high-intensity pulsed beam of metastable helium atoms, Meas. Sci. Technol. 11, 1510 (2000).

[29] D. M. Walker, A. A. Morgan, and S. D. Hogan, Cavity-enhanced Ramsey spectroscopy at a Rydberg-atomsuperconducting-circuit interface, Appl. Phys. Lett. 117, 204001 (2020).

[30] C. Overstreet, P. Asenbaum, and M. A. Kasevich, Physically significant phase shifts in matter-wave interferometry, Am. J. Phys. 89, 324 (2021).

[31] J. M. Hogan, D. M. S. Johnson, and M. A. Kasevich, Lightpulse atom interferometry, in Atom Optics and Space Physics, Proceedings of the International School of Physics "Enrico Fermi," Varenna, Italy, 2007, edited by E. Arimondo, W. Ertmer, W. P. Schleich, and E. M. Rasel (IOS Press, Amsterdam, 2009), p. 411.

[32] R. N. Zare, Angular Momentum (Wiley, New York, 1988).

[33] M. L. Zimmerman, M. G. Littman, M. M. Kash, and D. Kleppner, Stark structure of the Rydberg states of alkali-metal atoms, Phys. Rev. A 20, 2251 (1979).

[34] A. Deller and S. D. Hogan, Microwave spectroscopy of the $1 \mathrm{~s} n \mathrm{p}^{3} \mathrm{P}_{J}$ fine structure of high Rydberg states in ${ }^{4} \mathrm{He}$, Phys. Rev. A 97, 012505 (2018).

[35] K. Gawlas and S. D. Hogan, Rydberg-state ionization dynamics and tunnel ionization rates in strong electric fields, Phys. Rev. A 99, 013421 (2019).

[36] G. W. F. Drake, High precision theory of atomic helium, Phys. Scr. T83, 83 (1999).

[37] A. Deller and S. D. Hogan, Confinement of High- and Low-Field-Seeking Rydberg Atoms Using Time-Varying Inhomogeneous Electric Fields, Phys. Rev. Lett. 122, 053203 (2019).

[38] Y. Pu, D. D. Neufeld, and F. B. Dunning, Ionization of Rydberg atoms at metallic surfaces: Influence of stray fields, Phys. Rev. A 81, 042904 (2010).

[39] J. D. Carter and J. D. D. Martin, Energy shifts of Rydberg atoms due to patch fields near metal surfaces, Phys. Rev. A 83, 032902 (2011). 